\title{
Professor Dr. Heinrich Friedel zum 90. Geburtstag
}

\author{
Professor Dr. Heinrich Friedel on the Occasion of his $90^{\text {th }}$ Birthday
}

\section{Bibliografie}

DOI http://dx.doi.org/

10.1055/s-0029-1244118

Pneumologie 2010; 64:

320-321 @ Georg Thieme

Verlag KG Stuttgart · New York

ISSN 0934-8387

Korrespondenzadresse

Dr. med.

Jörg-Friedemann Fischer

Wallstr. 74

06484 Quedlinburg

friedemann.fischer@web.de
Heinrich Friedel wurde 1920 als Sohn eines Schlossers in Darmstadt geboren. Nach seinem Medizinstudium in Frankfurt/Main, Berlin und Breslau, das er 1944 mit dem Staatsexamen und der Promotion über „Die Verwendbarkeit von Schirmbild-Verfahren für Reihenuntersuchungen der Bevölkerung“ abschloss, wurde er zum Militärdienst an die Ostfront eingezogen und kam dort in Kriegsgefangenschaft, aus der er erst 1949 entlassen wurde.

Er nahm im Städtischen Krankenhaus Schwerin eine chirurgische Ausbildung auf, wechselte jedoch bald in die Tuberkulose-Heilstätte Schwerin-Lankow, die er 1952 als Lungenfacharzt verließ, um eine Oberarztstelle im neu gegründeten Tuberkulose-Forschungsinstitut in Berlin-Buch anzutreten.

Hier entwickelte Heinrich Friedel zusammen mit dem Ingenieur H. Becker (Fa. SECURA Berlin) das später nach ihm benannte Beatmungsbronchoskop mit proximaler Beleuchtung, das von nun an endoskopische Untersuchungen der Trachea und Bronchien in Relaxationsnarkose mit kontinuierlicher Ventilation ermöglichte und die zuvor geübte Narkosebronchoskopie in der zeitlich eng begrenzten Apnoetechnik ablöste. 1956 konnte das Beatmungsbronchoskop bereits auf der Leipziger Messe ausgestellt werden. Es entwickelte sich bald zu einem bedeutenden Exportartikel. Damit waren die technischen Voraussetzungen für die Ausarbeitung praxistauglicher, hocheffektiver endoskopischer Diagnostik- und Therapieverfahren geschaffen. An erster Stelle ist die Katheterbiopsie nach Friedel ${ }^{*}$ zu nennen. Als Therapieverfahren erlangten die Bronchustamponade bei lebensbedrohlichen endobronchialen Blutungen und die endobronchiale „Plombierung“ chronischer tuberkulöser Kavernen mit Antituberkulotika-Pasten große Bedeutung. In einer Zeit ohne Computertomografie lieferte die durch Friedel entwickelte Bronchografie in Relaxationsnarkose mit dem Doppellumentubus von E. Carlens

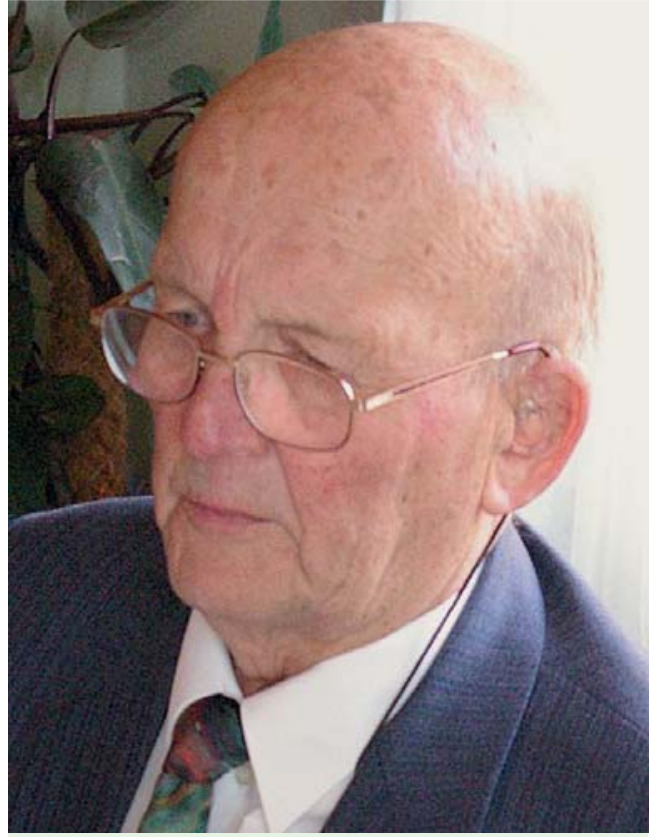

Professor Dr. Heinrich Friedel.

zur Darstellung des gesamten Bronchialbaumes eines vorgeblähten Lungenflügels in idealer anatomischer Position wichtige Informationen über Bronchiektasen vor einer geplanten Resektion sowie der bronchialen Beziehungen von Lungenherden und Hohlräumen als „Pfadfindermethode“ für deren endobronchiale Sondierung.

1958 übernahm Heinrich Friedel die Tuberkuloseheilstätte „Waldkrankenhaus Lostau“ als Ärztlicher Direktor und Chefarzt, die er in den folgenden Jahren zusammen mit seinen Mitarbeitern zu einer modernen Lungenklinik und zu einem Zentrum der Bronchologie entwickelte.

1960 habilitierte er sich an der Medizinischen Akademie Magdeburg mit seiner Arbeit „Die Katheterbiopsie des peripheren Lungenherdes“. Frühzeitig erkannte Friedel die Notwendigkeit einer Wiedererlangung akademischer Repräsen- 
tanz der Lungenheilkunde an der Hochschule und die Notwendigkeit enger Zusammenarbeit mit den medizinischen Einrichtungen der Region, besonders mit dem Krankenhaus Altstadt in Magdeburg unter Prof. Dr. K.-A. Koelsch und der Thoraxchirurgie in Vogelsang/Gommern unter MR Dr. G. Schoefer. Leider konnte die angestrebte Fusion mit der Thoraxchirurgie zu dieser Zeit nicht verwirklicht werden. Friedels Baupläne dazu waren nach 1990 jedoch die konzeptionelle Grundlage für die lange erstrebte Schaffung eines „kompletten“ Thoraxzentrums am Standort Lostau.

Bereits in den ersten Jahren scharte Heinrich Friedel eine große Zahl interessierter Ärzte und Naturwissenschaftler um sich, schuf Wohnraum und soziale Einrichtungen für seine Mitarbeiterinnen und Mitarbeiter und legte damit den Grundstein für die Entwicklung des „Waldkrankenhauses Lostau“ zu einem pneumologischbronchologischen Zentrum vor den Toren der Stadt Magdeburg. So entstand unter seiner Leitung der erste Herzkatheter-Arbeitsplatz im Bezirk Magdeburg, der nicht nur komplexen funktionsdiagnostischen Untersuchungen der kardiorespiratorischen Interaktionen, sondern auch rein kardiologischer Diagnostik diente. Ein bakteriologisch-mykologisches Labor und eine Abteilung für Asthma und Allergologie mit pneumologisch-arbeitsmedizinischem Schwerpunkt wurden aufgebaut. Als geradezu visionär sind Friedels Anstrengungen zu sehen, in der Mitte der 60er-Jahre in Lostau die endobronchiale Kleinraumbestrahlung maligner Tumoren zu verwirklichen.

Der Kampf gegen die Tuberkulose blieb auch nach dem Rückgang der Nachkriegsepidemie ein wichtiger Schwerpunkt in der Arbeit Heinrich Friedels. In besonderem Maße widmete er sich den sogenannten Chronikern mit nicht sanierungsfähigen Kavernen. Bei vielen dieser kardiopulmonal schwer geschädigten und somit funktionell inoperablen Menschen konnte er schließlich mit seiner bronchoskopischen Kavernenplombierung doch noch die für ein Leben in der Gesellschaft unabdingbare bakterielle Sanierung erreichen. Die bereits in seiner Promotion wissenschaftlich behandelten Schirmbildverfahren waren in seiner gleichzeitigen Verantwortung als Bezirkstuberkulosearzt zunächst das wichtigste Werkzeug zur Tuberkulose-Früherkennung im Rahmen der Volks-Röntgen-Reihenuntersuchungen (VRRU). In den folgenden Jahren wurde es auch zur Erkennung des Bronchialkarzinoms genutzt und von Heinrich Friedels Mitarbeitern in dieser Hinsicht auch wissenschaftlich evaluiert.

1962 verliehen der Rektor und der Senat der Medizinischen Akademie Magdeburg anlässlich des 60-jährigen Bestehens der Heilstätte dem „Waldkrankenhaus Lostau“ den Status der „Lungenklinik der Medizinischen Akademie Magdeburg“. Wenig später wurde Heinrich Friedel zum Professor mit Lehrauftrag Innere Medizin/Pneumologie ernannt und erhielt schließlich den ersten Lehrstuhl für Innere Medizin/Pneumologie an einer Hochschule der DDR.

Ein wichtiges Anliegen Heinrich Friedels war stets die Weitergabe seines fachlichen Wissens und seines großen praktischen Erfahrungsschatzes an Ärzte, Studenten und Mitarbeiter der anderen medizinischen Berufe. Das spiegelte sich nicht nur in der studentischen und ärztlichen Ausbildung an der Medizinischen Akademie Magdeburg wider, sondern auch in den vielen wissenschaftlichen Symposien sowie den alljährlichen bronchologischen Kursen, die in Lostau unter seiner Leitung hervorragende Referenten aus dem In- und Ausland, aus Ost und West, aus allen Fachgebieten, die bronchologische Methoden brauchten, zusam- menführte. Besonders aus den damaligen „Ostblockländern“ kamen viele Ärzte zum Erlernen moderner bronchologischer Techniken nach Lostau und profitierten dabei auch von den guten russischen Sprachkenntnissen Heinrich Friedels, die er während der Kriegsgefangenschaft erworben hatte und die ihm nun bei der Verbreitung seiner „bronchologischen Idee“ halfen. Anfangs engagierte sich Heinrich Friedel auch als pädiatrischer Bronchologe und liebte dieses Teilgebiet sehr. Er vertrat jedoch die Auffassung, dass Bronchologie bei Kindern möglichst von Kinderärzten praktiziert werden sollte. Mit Prof. Dr. W. Thal aus der Kinderklinik der Medizinischen Akademie Magdeburg fand er frühzeitig einen Schüler, der dieses Statement sehr erfolgreich umgesetzt hat.

1965 bis 1967 war Heinrich Friedel Präsident der „Gesellschaft für Lungenkrankheiten und Tuberkulose in der DDR“. Gleichzeitig betrieb er mit vielen Gleichgesinnten die Gründung einer „Gesellschaft für Bronchologie der DDR“, welche alle medizinischen Fachrichtungen vereinen sollte, die sich bronchologischer Untersuchungsmethoden bedienten und an ihrer Entwicklung arbeiteten. Die Befreiung der Bronchologie aus dem damals noch engen, „tuberkuloselastigen“ Korsett der „Gesellschaft für Lungenkrankheiten und Tuberkulose der DDR“ führte dazu, dass die Aufnahme in die „Association Internationale pour l'Étude des Bronches“ möglich wurde und Heinrich Friedel bereits 1970 deren XX. Congressus Bronchologicus in Dresden ausrichten durfte - zweifelsohne damals eine berufspolitische und organisatorische Großtat im „real existierenden Sozialismus“! Unbedingt erwähnt werden soll das frühzeitige Engagement Heinrich Friedels für die Rückführung der Lungenheilkunde in den Schoß der Inneren Medizin, um der modernen Pneumologie nicht nur die Beteiligung an universitärer Lehre und Forschung zu ermöglichen, sondern ihr auch wieder ausreichenden ärztlichen Nachwuchs zuzuführen. Mit Unterstützung des unvergessenen K.A. Koelsch gelang es ihm, in seiner Klinik schon frühzeitig Fachärzte für Innere Medizin mit pneumologischem Profil auszubilden.

Die Wertschätzung, die sich Professor Dr. Heinrich Friedel als Wissenschaftler und Hochschullehrer erwarb, wurde durch zahlreiche nationale und internationale Fachgesellschaften gewürdigt. Er ist Ehrenmitglied der „Gesellschaft für Bronchopneumologie und Tuberkulose der DDR“, der „Deutschen Gesellschaft für Pneumologie und Beatmungsmedizin“, der „Mitteldeutschen Gesellschaft für Pneumologie“ und des „Berufsverband der Pneumologen Sachsen-Anhalt“. Sein internationales Ansehen als Bronchologe hat 1998 auf dem 10. Weltkongress für Bronchologie in Budapest im Rahmen einer Ehrenvorlesung mit der Verleihung der Ehrenmitgliedschaft in der „World Association for Bronchology“ und der „Deszö-Kassey-Medaille“ die gebührende Anerkennung erfahren.

Seine ehemaligen Schüler und Mitarbeiter gratulieren dem Jubilar von Herzen und wünschen ihm auch in Zukunft alles erdenklich Gute, vor allem bestmögliche Gesundheit und weiterhin eine schöne gemeinsame Zeit in der Familie zusammen mit seinen Kindern, Enkeln und Urenkeln. Unsere dankbaren Erinnerungen gehen zurück in die Jahre, wo wir bei Heinrich Friedel lernen und arbeiten durften. Seine beruflichen Erfahrungen und väterliche Unterstützung haben uns immer begleitet und uns zu einem Teil der großen „Lostauer Familie“ werden lassen. Dafür sei ihm auch an dieser Stelle noch einmal gedankt. 